\title{
CONTASURE NEEDLELESS: TOT DE UNA SOLA INCISION PARA EL TRATAMIENTO DE LA INCONTINENCIA DE URINARIA DE ESFUERZO
}

\author{
Rafael Navazo, Jesús Moreno, Cristina Hidalgo, Miguel Angel Herraiz, José Antonio Vidart, \\ Jesús Salinas y Angel Silmi.
}

Unidad de Suelo Pélvico. Universidad Complutense. Hospital Clínico San Carlos de Madrid. Madrid. España.

\begin{abstract}
Resumen.- OBJETIVO: El objetivo de este estudio es describir la técnica quirúrgica, medir la seguridad, resultados y posibles complicaciones a medio plazo de una técnica sin agujas Contasure Needleless (Neomedic Internationall un TOT de una sola incisión para el tratamiento quirúrgico de la incontinencia urinaria de esfuerzo.
\end{abstract}

Un concepto básico de esta malla es que no es un Minislings, el Contasure Needleless es un TOT de una sola incisión posee una superficie de fibrosis 138\% mayor que los minisling. Analizarémos el resultado de este procedimiento mínimamente invasivo. El área de soporte uretral del Contasure Needleless es muy similar al TOT (un 16\% menor). El material es 100\% polipropileno macro poroso.

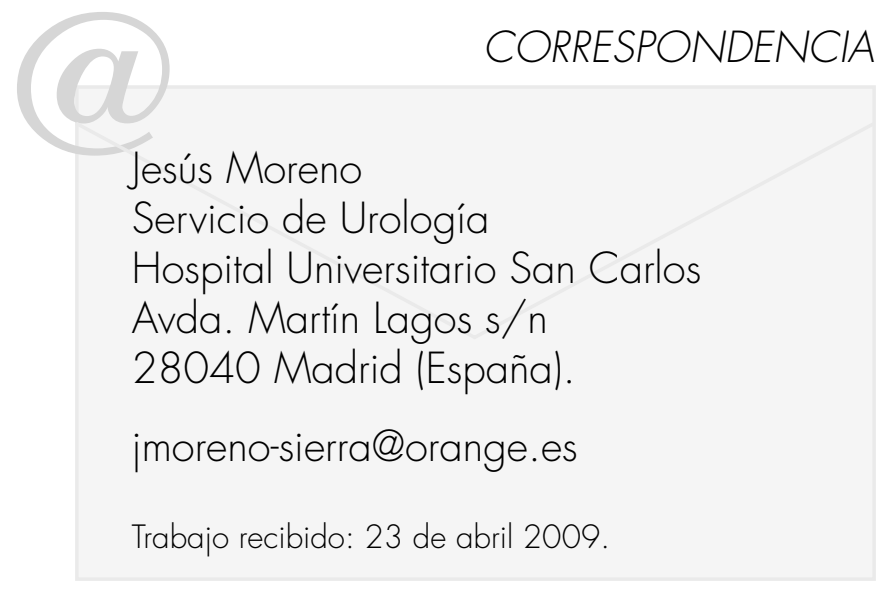

MÉTODO: 120 pacientes fueron evaluadas retrospectivamente. Todas las pacientes fueron operadas por incontinencia urinaria de esfuerzo con Contasure Needleless. El protocolo clínico que se siguió en todas estas pacientes fue test de esfuerzo, estudio urodinámico y cuestionario de calidad de vida antes y después del procedimiento. El criterio de inclusión fueron pacientes con incontinencia urinaria de esfuerzo en muchos casos con cirugía asociada. Pacientes con déficit intrínseco del esfínter o incontinencia neurógena fueron excluidas del estudio. La anestesia fue general en un 30\% de las pacientes y epidural en un $70 \%$.

Se coloco una malla de polipropileno libre de tensión en la uretra media de $114 \mathrm{~mm}$ de largo (Contasure Needleless). La parte central la malla tiene $12 \mathrm{~mm}$ ancho. La Malla puede ser ajustada durante la cirugía, posee dos bolsillos a cada lado. Estos bolsillos ayudan en un primer momento a mantener la malla y mas adelante proporcionaran un soporte a la malla debido a la fibrosis. Se hace una incisión suburetral de $20 \mathrm{~mm}$ y se disecan los espacios parauretrales hasta la rama isquiopubica. Una Bengolea se introduce en los bolsillos y se cierra, después se inserta en los espacios disecados hasta penetrar el musculo obturador interno abriendo el bolsillo una vez penetrado el musculo, los mismos pasos se realizan en el lado contrario.

RESULTADOS: 120 pacientes con una edad media de 55 años (36 a 79) y una media de 3 partos (0-7). Seguimiento medio: 24 meses. De los 120 pacientes 100 de ellos (84\%) están totalmente curados, Mejorados 10 pacientes (8\%) y fracasos un 10 (8\%). El tiempo medio de operación de colocación de la malla fue de 9 minutos (4-12). Complicaciones: 3 pacientes tuvieron una extrusión vaginal de la malla resuelta con estrógenos y 2 pacientes tuvieron dificultades temporales de vaciado, ningún paciente sufrió de dolor inguinal.

CONCLUSIONES: Los resultados del estudio sugieren que el Contasure Needleless puede ser considerado un 
tratamiento de elección para tratamiento quirúrgico de la incontinencia urinaria de esfuerzo. Es un tratamiento mínimamente invasivo, sin el uso de agujas y mantiene resultados similares de curación a dos años de seguimiento que el TOT convencional.

Palabras clave: Incontinencia Urinaria de Esfuerzo. TOT con una sola incisión.

Summary.- OBJECTIVES: The aim of this study is to describe the surgical technique, and assess the complications and middle-term results of the Contasure Needleless (Neomedic International), a single incision TOT sling for the surgical treatment of stress urinary incontinence.

The main concept of this device is that it is not a minisling, it has $138 \%$ more surface area. We are analyzing the results of a minimally invasive solution that is a TOT like sling with the same known benefits of a TOT and the advantages of a single incision technique. The surface area to support the urethra of the Needleless is very similar to the surface area of the TOT. $116 \%$ less surface area of tissue ingrowths) It is 100\% macroporous polypropylene without any additional material.

METHODS: 120 patients were evaluated retrospectively. They were all treated of SUI with the Contasure Needleless. Female patients were evaluated under clinical study protocol consisting in cough test, urodynamic and Quality Of Life questionnaire, before and after the procedure. Inclusion criteria: patients with genuine SUI and patients with SUI plus concomitant procedures as prolapse. Exclusion criteria: patients with ISD and or neurogenic incontinence. Anesthesia used: general (30\%) or epidural (70\%), patients with associated pathology.

PROCEDURE: The $114 \mathrm{~mm}$ long and tension-free mesh was placed beneath the midurethra. The central part is $12 \mathrm{~mm}$ wide. The sling can be repositioned during surgery due to the $22 \mathrm{~mm}$ wide T-Pocket Positioning System located at the 2 edges. These pockets fixed the sling to the surrounding tissue in order to have the proper tissue in growth and anchoring. A $20 \mathrm{~mm}$ sub urethral incision was made to dissect the paraurethral spaces only up to the ischiopubic ramus. A surgical forceps with the T-pocket folded was inserted into the dissected spaces and penetrates at the contra lateral side, like the standard transobturator technique. The forceps was introduced until the fascia of the Internal Obturator muscle was perforated. Then the forceps is opened to extend the pocket inside the muscle fibers for fixation.

RESULTS: 120 patients with a mean age of 55 years (from 36 to 79) and a mean number of deliveries of 3 (0-7). Follow-up period: 24 months. Success rate: 100 patients (84\%) Improved rate: 10 patients (8\%) Failure rate: $10(8 \%)$ Mean operating time of sling procedure alone: 9 minutes (4-12). Complications: 3 patients had mesh extrusion solved with estrogens and 2 patients required short term catheterization due to voiding difficulties and no patients had inguinal pain.

CONCLUSIONS: The results of the study suggest that the Contasure Needleless can be considered a minimally invasive TOT with no-needles and maintaining the same cure rate than our TOT cases at 2 years follow up.

Keywords: Stress Urinary incontinence. Single Incision TOT.

\section{INTRODUCCIÓN}

Existen diferentes factores que debemos considerar en el desarrollo de IUE: embarazos y multiparidad, menopausia, diabetes, obesidad, envejecimiento, etc. (2-3). De esta forma se inducen cambios en los mecanismos de soporte uretrales y/o en el mecanismo esfinteriano (3). A pesar de haberse descrito numerosas técnicas quirúrgicas para su tratamiento, no existe una única intervención capaz de resolver todos los casos que se pueden presentar en la mujer. Por tanto antes de tomar la decisión de qué tipo de técnica es la que creemos más idónea, deberemos evaluar cuidadosamente las características clínicas y urodinamicas de la paciente, así como las preferencias y habilidad del cirujano (4). La colposuspensión retro púbica descrito por Burch en 1961 (5), es el procedimiento que a demostrado tener un mayor porcentaje de éxito a corto y largo plazo, con éxito en el $85-90 \%$ a los 5 años y $70 \%$ de continencia durante los 5 años siguientes (6). A pesar de estos buenos resultados, la técnica de Burch tiene efectos secundarios como problemas para vaciar la vejiga, "de novo" urgencia o prolapso anterior en un $20 \%$ de los casos (7).

Siguiendo los principios de la teoría integral de IUE, desarrollada por Petrus y Ulmsten, el mecanismo de la incontinencia está basado en una combinación de estructuras anatómicas que se integran en una compleja coordinación para abrir y cerrar el cuello de la vejiga y la uretra (8). Basado en este modelo, en 1995, Ulmsten desarrollo una nueva técnica basado en una malla de polipropileno para soportar la uretra sin tensión (TVT®) (9).

Las técnicas vaginales sin tensión han conseguido resultados similares a los descritos con la técnica de Burch con la ventaja de ser una cirugía de mínima invasión con menos morbilidad y por estos motivos y con el apoyo de los resultados publicados, podemos considerar a las técnicas libre de tensión como procedimientos que ofrecen los mismos resultados a corto y largo plazo (10-12). 
Las complicaciones más frecuentes de esta técnica son la consecuencia del paso de las agujas para colocar la malla. Perforación de la vejiga (0$23 \%$ ) o Hematoma de Retzius (0,8-3,3\%). También se describen otras complicaciones como la perforación del intestino, lesiones vasculares y nerviosas (13).

Basado en la técnica original, Delorme desarrolla una nueva técnica pasando la aguja vía el foramen del musculo obturador, evitando pasar las agujas por el espacio retro púbico (14) para no perforar la vejiga y tampoco crear un hematoma en la zona Retzius reduciendo el tiempo de la cirugía al no tener que utilizar el cistoscopio (15). Jean Leval modifico aun mas esta técnica pasando las agujas desde la vagina hacia el musculo obturador (16) para evitar problemas vasculares y neurológicos relacionados al paso de las agujas por esta zona. Los resultados iníciales fueron similares a la técnica retro púbica pero con un menor riesgo tanto durante la cirugía como en el postoperatorio (17). Todo esto ha llevado a un descenso importante en el índice de complicaciones debido a esta técnica.

\section{OBJETIVO}

Con el objetivo de simplificar las técnicas anteriores y minimizar las posibles complicaciones relacionadas con el paso de las agujas una nueva técnica ha sido desarrollada manteniendo el concepto de libre de tensión e introduciendo el concepto de Contasure Needleless (Sin Agujas). La malla se coloca sin la necesidad de agujas, manteniendo la malla debajo de la uretra media como una hamaca (Contasure Needleless Technique ${ }^{\circledR}$ ).

El objetivo de este estudio es describir la técnica quirúrgica, medir la seguridad, resultados y posibles complicaciones a medio plazo de una técnica sin agujas para el tratamiento quirúrgico de la incontinencia urinaria de esfuerzo.

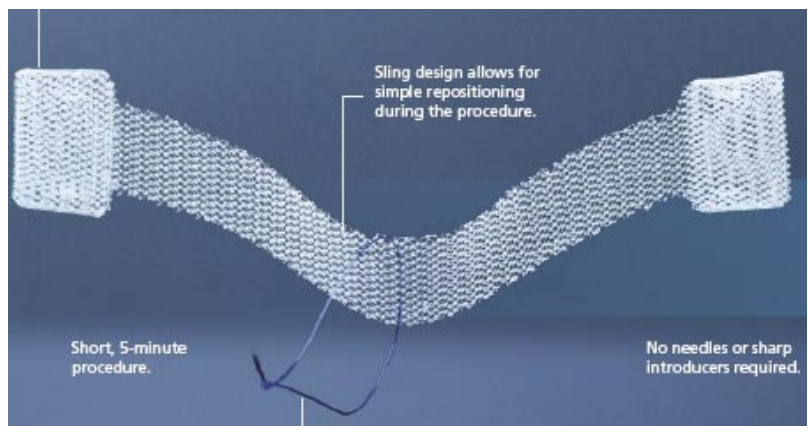

FIGURA 1

\section{MATERIAL Y MÉTODO}

En la Unidad de Suelo Pélvico del Hospital Clínico San Carlos, hemos realizado un estudio retrospectivo sobre 120 pacientes, durante el periodo comprendido entre Marzo 2006 y Septiembre 2008. En todas las pacientes se realizó una historia y exploración física completa, test de calidad de vida ICI-Q y un estudio urodinámico completo.

Todos los pacientes presentaban IUE por hipermovilidad de la uretra desde moderada a severa.

La malla Needleless está fabricada en Polipropileno monofilamento de $0,4 \mathrm{~mm}$ de diámetro, macroporo (Amid I) y una porosidad del $55 \%$ con unas dimensiones de $114 \times 12 \mathrm{~mm}$. Sus extremos son más anchos $(22 \mathrm{~mm})$, y un bolsillo que ayuda a su colocación y sujeción de la (Figura 1). Estos bolsillos son la mayor ventaja de las mallas.

Analizamos edad, paridad, cirugía asociada, grado de incontinencia, tiempo de la cirugía, complicaciones durante y postoperatorias y continencia a corto plazo

\section{TÉCNICA}

La técnica puede realizarse con cualquier tipo de anestesia, preferiblemente local o regional. Se coloca a la paciente en posición de litotomía con las piernas flexionadas y se procede al vaciamiento vesical con un catéter Foley. Posteriormente realizamos una incisión longitudinal $(1-2 \mathrm{~cm})$ sobre se la mucosa vaginal debajo $(0,5 \mathrm{~cm})$ del meato uretral y se disecan los espacios para uretrales hacia ambos lados (a las 10 y a las 2 en punto) hasta que tocamos la rama descendente de la rama isquiopubiana. Un fórceps (Kocher o Bengolea) se coloca dentro del bolsillo de la malla Needleless haciendo una hiper

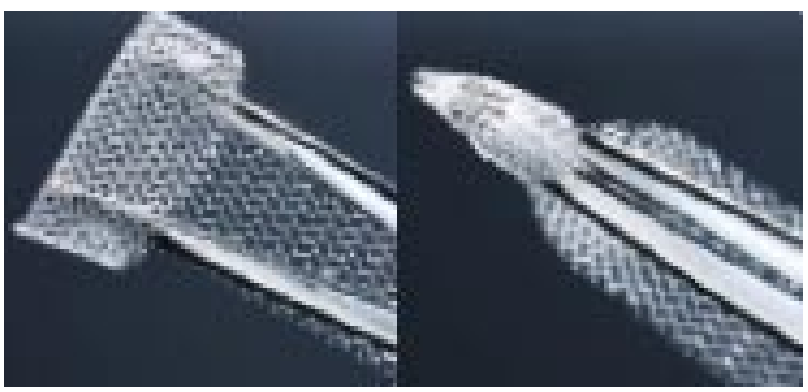

FIGURA 2. 
extension y volviendo a cerrar la malla como puede verse en la Figura 2.

El fórceps con la malla doblada se introduce en el espacio para uretral y el musculo obturador interno se perfora (Figura 3).

A continuación se procede a la apertura del fórceps para extender la posición del bolsillo en forma de T (Figura 4), para posteriormente proceder al cierre y extracción de la vagina, dejando fija la malla Needleless.

Para controlar la inserción de la punta del fórceps y de la malla en el musculo obturador interno el cirujano puede sujetar la malla por el hilo central conectado a la malla. La misma maniobra se realiza en la dirección contraria para terminar la inserción de la malla.

Una vez la malla es introducida se puede recolocar introduciendo de nuevo el fórceps en los bolsillos de la malla empujando un poco más. Para disminuir la presión de la malla sobre la uretra el cirujano puede tirar un poco del hilo central y liberar tensión. Cuando la malla está perfectamente colocada en contacto con la uretra el hilo central será cortado y tirando de él este será completamente retirado. La incisión vaginal será cerrada con una sutura absorbible de $2 / 0$.

El catéter uretral se mantendrá hasta que el efecto de la anestesia haya desaparecido y la paciente será dada de alta una vez comprobado que el residuo después de la micción es menor a 100 cc.

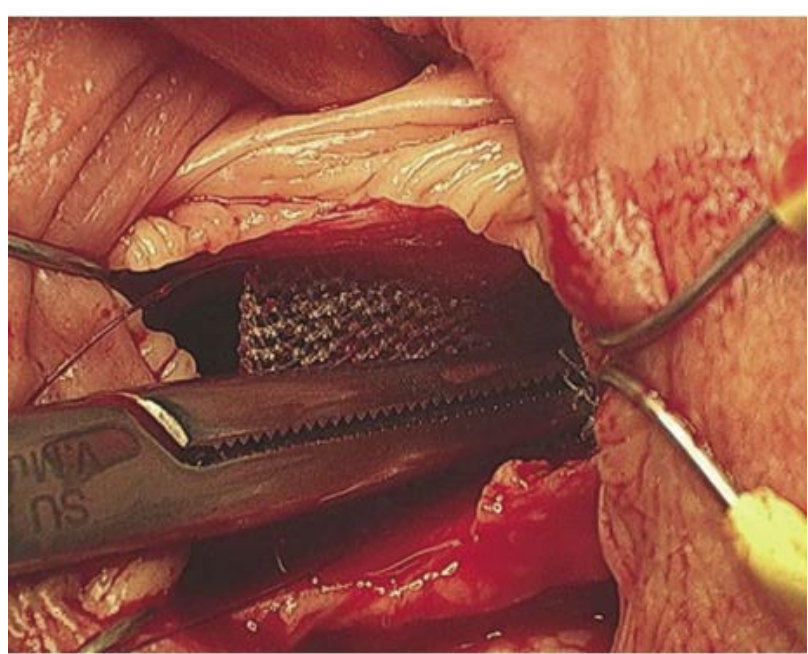

FIGURA 3

\section{RESULTADOS}

Los siguientes parámetros se han analizado para los primeros 120 pacientes: edad media, partos vaginales, años de evolución del IUE, cirugía asociada, tiempo medio de cirugía, cateterización uretral postquirúrgica, complicaciones inmediatas postoperatorio.

La edad media de los pacientes era de 50 años (40-70), media de partos vaginales 3 (1-4). Tiempo medio de incontinencia antes de la intervención: 10 años (1-30).

Todos los pacientes presentaban síntomas de incontinencia que fueron confirmados mediante un estudio urodinámico. La media del test de Sandvik fue de 8 (3-11). En nuestra serie se asocio a la técnica una reparación vaginal anterior en 70 pacientes y en 30 pacientes se les realizo una histerectomía en la misma intervención. El resto no tenían ningún prolapso asociado.

El tiempo medio de cirugía fue de 9 minutos (4-12) y la estancia media fue de 1,5 días (1-5) teniendo en cuenta que las pacientes con 2 días de estancia son las que fueron intervenidas por un cistocele III o IV.

Durante el postoperatorio inmediato 2 pacientes sufrieron retención que se resolvió con cateterización. En esta paciente se realizo una reparación de la vagina anterior. No detectamos casos de dolor inguinal, hemorragias, hematomas, infección urinaria o daño vascular o visceral.

Después de un periodo de seguimiento medio de 24 meses (18-32), 100 pacientes (84\%) están objetivamente curados de incontinencia (test de Sandvik y test de estrés son negativos). 10 pacientes

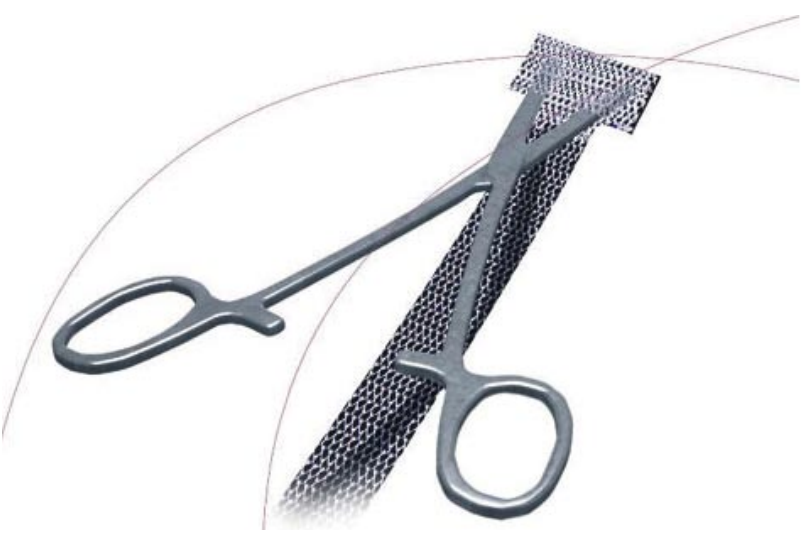

FIGURA 4 
TABLA I.

\begin{tabular}{|l|c|}
\hline & Porcentajes \\
\hline Curación & $100(84 \%)$ \\
\hline Mejoría & $10(8 \%)$ \\
\hline Fracasos & $10(8 \%)$ \\
\hline Seguimiento medio & 24 meses \\
\hline
\end{tabular}

muestran mejoras $(8 \%)$ y $10(8 \%)$ se consideran fallos (Tabla I).

Complicaciones a largo plazo incluyen tres casos de extrusión de la malla $(0,5 \mathrm{~cm})$ que se solucionaron con estrógenos.

\section{CONCLUSIONES}

La técnica Contasure Needleless proporciona un nuevo concepto para tratar el IUE con una malla libre de tensión y debido a su fácil implantación y bajo índice de complicaciones cumple con los criterios de cirugía de mínima invasiva.

\section{BIBLIOGRAFÍA y LECTURAS RECOMENDADAS (*lectura de interés $y^{* *}$ lectura fundamental)}

1. Moreno Sierra J, Galante I, Prieto S, Fernandez L, Silmi A. Conceptos y terminologia utilizada en incontinencia urinaria y prolapsos urogenitales. Moreno Sierra, J y Silmi Moyano, A. Atlas de Incontinencia Urinaria y Suelo Pélvico ENE Ed. 2006; 77-81.

2. Moreno Sierra J, Galante I, Prieto S, Fernández L, Silmi A. Etiología, patogenia, tipos y clasificaciones de incontinencia urinaria y prolapsos urogenitales. Moreno Sierra, J y Silmi Moyano, A. Atlas de Incontinencia Urinaria y Suelo Pélvico ENE Ed. 2006; 83-100.

3. Wahle G, George P, Young H, Raz S. Anatomy and Patophisiology of pelvic support. Raz Female Urology 2nd Ed. WB Saunders Company 1983; 57-72.

*4. Moreno Sierra J, Galante I, Prieto S, Fernández L, Silmi A. Etiología, patogenia, tipos y clasificaciones de incontinencia urinaria y prolapsos urogenitales. Moreno Sierra, J y Silmi Moyano, A. Atlas de Incontinencia Urinaria y Suelo Pélvico ENE Ed. 2006; 477-81.
5. Burch JC. Urethrovaginal fixation to Cooper's ligament for correction of stress incontinence, cystocele and prolapse. Am. J. Obstet. Gynecol. 1961; 81:281-90.

6. Lapitan MC, Cody DJ, Grant AM. Open retropubic colposuspension for urinary incontinence in women. (Cochrane review). Cochrane Library, issue 1. Oxford: update software, 2003.

7. Wiskind AK, Creighton SM, Stanton SL.The incidence of genital prolapse after Burch colposuspension. Am. J. Obstet. Gynecol. 1992; 167:399-405.

*8. Petros PE, Ulmsten UI. An integral theory of female urinary incontinence: experimental and clinical considerations. Acta Obstet. Gynecol. Scand. Suppl. 1990; 153:7-31.

*9. Ulmsten U, Henriksson L, Johnson P, Varhos G. An ambulatory surgical procedure under local anesthesia for treatment of female urinary incontinence. Int. Urogynecol. J. 1996; 7:81-6.

10. Ward K, Milton P. United Kingdom and Ireland Tension-free Vaginal Tape Trial Group. Prospective multicentre randomised trial of tension-free vaginal tape and colposuspension as primary treatment for stress incontinence. Br. Med. J. 2002; 41:469-73.

11. Gutiérrez Baños JL, Martín García B, Portillo Martín JA, Del Valle Schaan JI, Hernández Rodríguez R, Correas Gómez MA, et al. Tratamiento de la incontinencia urinaria de esfuerzo mediante el implante suburetral de cintas libres de tensión (TVT). 4 años de experiencia. Arch.Esp. Urol. 2003; 55(8):885-92.

**12. Nilsson CG, Falconer C, Rezapour M. Seven-year follow-up of the tension-free vaginal tape procedure for treatment of urinary incontinence. Obstet. Gynecol. 2004; 104:1259-62.

13. Ward KL, Hilton P. A prospective multicenter randomized trial of tension-free vaginal tape and colposuspension for primary urodynamic stress incontinence: two-year follow-up. Am. J. Obstet. Gynecol. 2004; 190(2):324-31.

14. Boustead GB. The tension-free vaginal tape for treating female stress urinary incontinence. BJU Int. 2002; 89:687-93.

15. Delorme E. Trans-obturator urethral suspensión: a minimally invasive procedure to treat female stress urinary incontinence. Progr. Urol. 2001; 11:1306-13.

16. Delorme E, Droupy S, De Tayrac R, Delmas V. Trans-obturator tape Uratape ${ }^{\circledR}$, a new minimally invasive treatment for female urinary incontinence. Progr. Urol. 2003; 13:659-9.

17. Pardo Schanz J, Ricci Arriola $P$, Solà Dalenz V, Tacla Fernández X. Experiencia en la corrección quirúrgica de la incontinencia de orina de esfuerzo con técnica TOT (Trans-Obturator-Tape). Arch. Esp. Urol. 2006; 59(3):225-32. 